\title{
Se doper via Internet ? Un jeu... de souris !
}

\section{Doping via the Internet? ... a mouse game!}

Véronique DUMESTRE-TOULET

Laboratoire BIOffice, avenue Gay Lussac - 33370 ARTIGUES PRES BORDEAUX Tél : 0556407382 - Fax : 0556407381

(Reçu le 2 décembre 1999 ; accepté le 27 décembre 1999)

\section{RÉSUMÉ}

Le but de cet article est de montrer au lecteur la facilité déconcertante avec laquelle on peut se doper via Internet. Les produits que l'on trouve concernent le dopage sportif, mais également intellectuel, sexuel, la lutte anti-âge et même le dopage animal..

"les bonnes adresses" ont été recherchées via les moteurs de recherche classiques. Elles ne sont pas exhaustives car le nombre de sites où l'on peut acheter des stéroïdes anabolisants ou leurs précurseurs, de l'hormone de croissance, de l'insuline, des beta-adrenergiques ou bien encore du Viagra ${ }^{\circledR}$, ou du Prozac ${ }^{\circledR}$, est impressionnant.

Plus de 200 sites web ont été visités lors de la réalisation de ce travail. Ils proposent à l'Internaute l'achat sécurisé des substances, mais aussi des conseils d'utilisation, les cures avant, pendant et après les compétitions, des compléments alimentaires, des témoignages d'utilisateurs et des ouvrages spécialisés.

L'acheteur n'a cependant aucune garantie sur la qualité du produit livré comme en témoigne la liste des contrefaçons publiée sur certains sites et supportera toute responsabilité vis à vis de l'importation de substances réglementées dans certains pays.

Cet article souhaite aujourd'hui rappeler que si Internet est un outil formidable, il représente aussi un danger en facilitant certaines pratiques, sans aucun contrôle possible, dont celles du dopage.

\section{MOTS-CLÉS}

dopage, Internet, anabolisants.

\section{SUMMARY}

This papers reviews the possibilities of buying performance enhancing drugs through Internet.

The drugs are proposed for sport, sexual behaviour, brain stimulation, to look young or to increase animal weight. Links were established by typical Internet search engine.

Daily, new addresses are added, most of them involving anabolics, growth hormone, insuline, $\beta$-adrenergics, Viagra or Prozac.

About 200 web pages have been tested during this study. Secured payment by credit card, health informations, use advises, drug combinations, specialised books and athletet report can be found. The purchaser has no warranty of quality, as a lot of fraudulent imitations have been reported. Moreover, he will be responsible for the import permission, particularly in case of restricted substances.

This paper demonstrate that Internet is a powerful tool, with major health risks when abused. 


\section{Introduction}

L'homme a toujours cherché à augmenter ses performances physiques et psychiques. Les techniques de dopage sont en constante évolution.

Les voies traditionnelles du dopage font appel à des prescriptions médicales pour les médicaments ou nutriments utilisés et commercialisés dans le circuit pharmaceutique.

Se procurer des substances dopantes est cependant difficile par le circuit classique pour les non initiés et souvent onéreux. Beaucoup de produits sont interdits en France mais en vente libre dans d'autres pays.

Internet propose de manière tout à fait aisée la vente de nombreux produits. Les sites sont accessibles à tous et plus de 200 ont été répertoriés grâce aux moteurs de recherche classiques.

On trouve ainsi des stéroïdes comme la testostérone ou la nandrolone, l'androstènedione ou diol, la 19 norandrostènedione ou diol, la DHEA, de la créatine, de la melatonine, de l'hormone de croissance, de l'insuline, de nombreux complexes vitaminiques et protéines etc...à l'usage des sportifs, mais aussi des dopants sexuels et des dopants intellectuels par le biais de médicaments psychoactifs, vendus "over the counter"

Certains sites conseillent le type de produit à choisir, le mode d'emploi en fonction du moment de la compétition pour les sportifs, les régimes à adopter, de même pour les substances psychoactives, les "élixirs de jouvance" ou les stimulants sexuels.

Des ouvrages très complets sur ces sujets sont également proposés.

Les prix sont attractifs et dégressifs en fonction de la quantité commandée. Le paiement est sécurisé par carte bancaire et la livraison très rapide par les transporteurs classiques. Des assurances quant à une non réception éventuelle sont aussi proposées.

L'objet de cet article est de vous donner un aperçu de ces sites, des produits que l'on y trouve, des conseils que l'on y prodigue et des résultats que l'on peut obtenir.

\section{I - Les produits proposés, adresses et ouvrages}

\section{Le dopage du sportif}

A tout seigneur, tout honneur, c'est en effet aux produits à l'intention des sportifs et en particulier aux bodybuildés que l'Internet consacre le plus grand nombre de pages, dont une sélection, non exhaustive est présentée ici.
- Les stéroïdes anabolisants, précurseurs ou stimulants de leur production

La nandrolone, les différents esters de testostérone (undecanoate, cypionate, enanthate, propionate..), la fluoxymesterone, la mesterolone, le stanozolol, la methenolone sont en vente sur les sites VHP vitality health products et PHARMASIA performance enhancement distributors, logos des firmes pharmaceutiques correspondantes à l'appui :

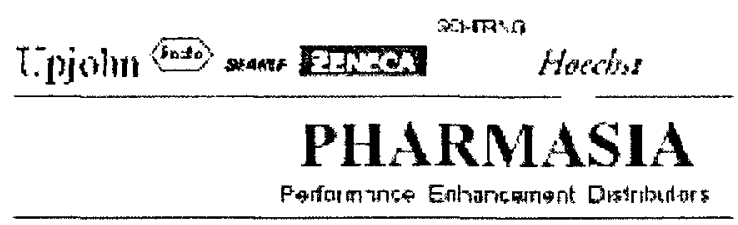

- http://www.feral.org/vitality/active.htm

- http://myhome.shinbiro.com/ pharmasi/index.html

Androsténedione, Androstènediol, précurseurs (ou prohormones) et métabolites de la testostérone ; 19-norandrostenedione ou norandrostenediol précurseurs de la nortestosterone ou nandrolone, sont proposés seuls ou associés et à différents dosages sur plusieurs sites comme muscleshop.net, massquantities.com, healthkick.net, price-power.com, vermontnutition.com, milleniumfitness.com, bigbodies.com ou encore americanmuscle.com. Ces sites proposent aussi hormone lutéinisante (LH ou tribulus terrestris) pour stimuler la production de testostérone, dehydroepiandrostérone (DHEA), hormone de croissance $(\mathrm{GH})$ et facteur de croissance (IGF 1A).

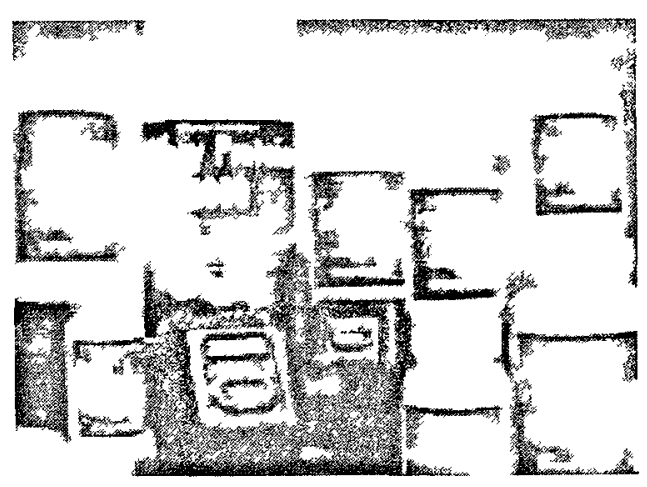

- http://www.muscleshop.net

- http://www.shop.massquantities.com

- http://www.healthkick.net/andro.htm

- http://www.prices-power.com

- http://www.arnutrition.com/

- http://www.milleniumfitness.com

- http://www. vermontnutition.com

- http://www.bigbodies.com

- http://www.americanmuscle.com/

- http://www.growthhormoneproducts.com/growthhormone.html 


\section{II - Conseils d'utilisation et Résultats}

Comme acheter ce type de substances ne sert à rien si on ne sait pas comment les utiliser, certains sites conseillent les produits à choisir selon la compétition, les combinaisons et le programme posologique optimal en fonction du moment de celle-ci.

Si l'internaute a encore des doutes, il peut acheter via le net des ouvrages comme THE STEROID BIBLE ou s'abonner on-line à des magazines comme TESTOSTERONE Magazine. Il y apprendra où se procurer les substances "légalement", comment utiliser les stéroïdes et éviter les effets secondaires ou comment passer les contrôles antidopages... Les témoignages et expériences de sportifs sont sollicités voire récompensés d'une prime s'ils sont retenus pour la publication mensuelle du site Roids.com

-http:// www.roids.com

-http://www.t-mag.com/

Les résultats sont présentés sur différents sites, où l'on peut également acheter posters et vidéos des culturistes photographiés.
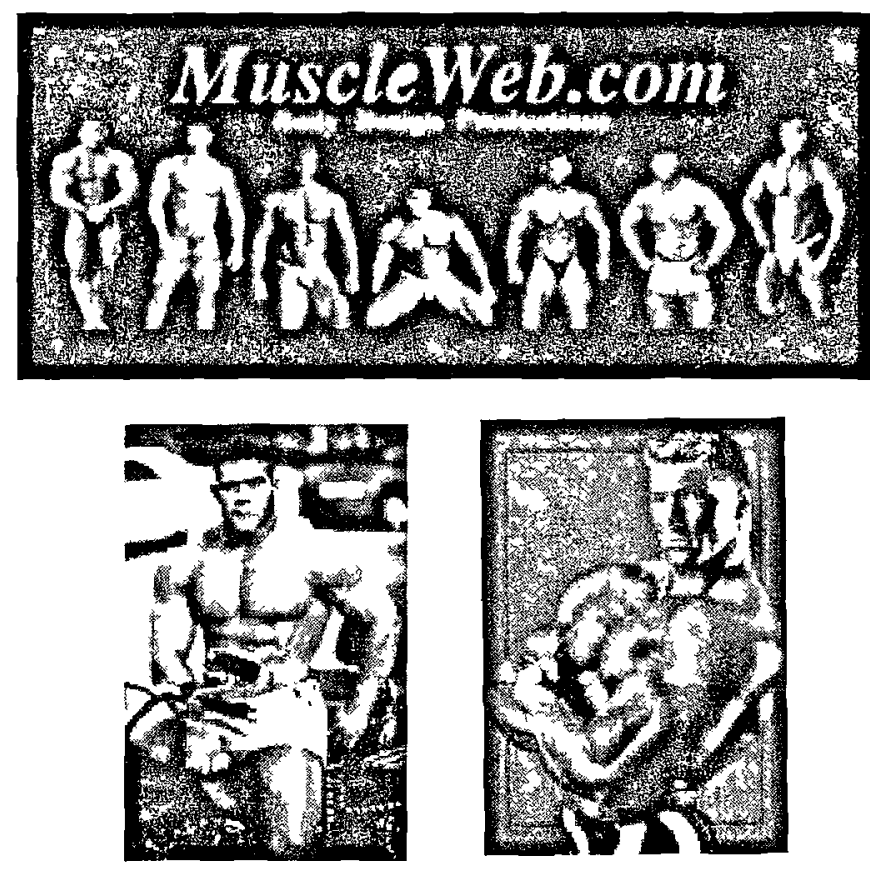

-http://muscleweb.com/

-http://www.bigbodies.com/BBpage1.html

\section{- Le clenbuterol et les beta adrenergiques}

On peut les acheter tout aussi aisément sur les sites Pharmasia, At cost Rx drugs ou E-pills

- http://myhome.shinbiro.com/ pharmasi/index.html

- http://albuterol.proventil.ventolin.atrovent.gen-next.com/

- http://www.epills.com/store/rxbrowse
- Les substances amaigrissantes (fat-burners) et /ou diurétiques

Muscleshop.com ou massquantities.com proposent un précurseur de l'hormone thyroidienne T3 : l'acide triiodothyroacetique ou TRIAC ainsi que des associations de caféine, norephedrine ou ephedrine et yohimbine, le site healthkick un diurétique (taraxatone) pour augmenter la définition musculaire, réduire la rétention hydrique et augmenter la vascularité lors des compétitions.

Sur le site Health central Rx , on trouve de l'Insuline sous différentes formes et dosages.

- http://shop.massquantities.com/cgi-bin/Mass

Quantities.storefront/1772960534/Product/View/115

- http://www.nutrition-city.com/talk_tips /metabolic thyrolean.htm

- http://www.muscleshop.net/Tiratricol.htm

- http://healthkick.net/taraxatone.htm

- http://www.epills.com/store/rxbrowse

- http://www.prices-power.com/fatburners.htm

- Pour lutter contre les éventuels effets secondaires...

Les effets secondaires produits par les stéroïdes comme certains troubles hormonaux, atteintes hépatiques et prostatiques, calvitie, acné sont bien connus et on peut trouver aux adresses suivantes des produits type flavonoïdes : Ipriflavone, Methoxy isoflavone qui augmentent la synthèse protéique sans produire ce type d'effets, des produits comme le minoxidil ou le finastéride pour lutter contre l'alopécie, des antiacnéiques voire des oestrogènes, des protecteurs veineux comme les extraits de Ginko Biloba.

- http://shop.massquantities.com/cgi-bin/

MassQuantities.storefront/1577315923/Product/

View/105

- http://www.prices-power.com/chrysin.htm

- http://www.feral.org/vitality/active.htm

- http://www.drugs-express.com/a/10070/propecia.html

- http://www.ionnet.com/ginko.html

\section{- La créatine}

L'absence de législation et d'interdiction concernant l'emploi de la créatine dans le domaine sportif en fait un produit à la mode, voire promotionnel sur beaucoup de sites Internet. Cette substance très prisée pour améliorer la performance athlétique sur un court terme, peut parfois être obtenue. gracieusement avec l'achat d'autres substances.

- http://www.prices-power.com/ creathp.htm

- http://www.vitawise.com/ dheacrea.htm

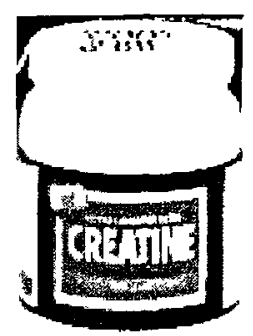




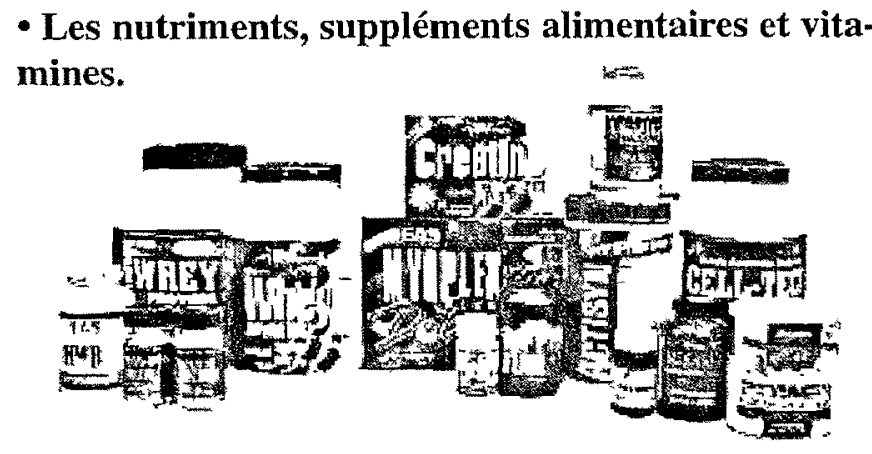

Des compléments vitaminiques, des minéraux, des barres repas ou milk-shakes hyperprotéinés, des gélules de plantes et extraits végétaux s'achètent sur la plupart des sites s'adressant aux sportifs et en particulier aux adresses suivantes :

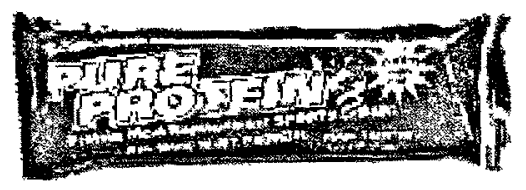

- http://members.tripod.com/VitaNherbs/index-8.html

- https://earth.galstar.com/kicker-cgi/osborn/web _store.cgi?product=vit01 \&cart_id=9032048.10265

- http://portland.citysearch.com/E/V/PDXOR 10002/97/73/9.html

\section{Les élixirs de jouvence}

"Prendre de l'âge est un mal qui se soigne" apprend t'on sur le site antiaging-research.com. Plusieurs sites proposent des substances dans le cadre d'un programme de lutte contre le vieillissement : hormone de croissance, DHEA, pregnenolone, melatonine, médicaments contre l'arthrose, sélénium et antioxydants comme les picnogénols sont actuellement les produits phares de cette lutte.

- http://www.antiagingresearch.com/

\section{La DHEA :}

La DHEA, est un neurostéroide utilisé également par les athlètes dont le taux diminue de façon importante après 25 ans. Des études sur l'animal ont montré l'intérêt de la supplémentation par la DHEA pour lutter contre certains effets du vieillissement, sur le plan physique mais aussi cérébral et immunitaire. Plusieurs sites proposent dans ce cadre de la DHEA aux adresses suivantes :

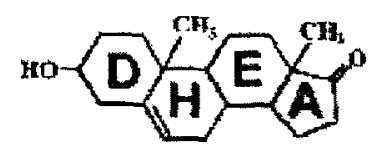

Dehydroepiandrosterone The Fountain of Youth
- http://www.vitawise.com/dheacrea.htm

- http://www.prices-power.com/dheaa.htm

- http://www.ionnet.com/dhea.html

\section{L'hormone de croissance}

Elle diminue la masse graisseuse, le cholesterol, la tension arterielle, régénère la masse osseuse et musculaire, la mémoire et la fonction autoimmunitaire...l'hormone de croissance est à l'honneur sur nombre de sites : - http://www.growthhormoneproducts.com/growthhormone.html

- http://www.anti-aging,org/

- http://www.secretagoguehgh.com/

- http://hgh7000.com/index.html

- http://www.ageless.com/index.html

\section{La pregnenolone}

Parce que la pregnenolone stimule la production de DHEA, diminue le stress et renforce les connexions neuronales, des sites la vendent pour lutter contre les méfaits de l'âge :

- http://www.prices-power.com/preg.htm

- http://www.feral.org/vitality/active.htm

\section{Divers}

Aux adresses suivantes, l'internaute pourra se procurer des antirhumastimaux, des produits pour lutter contre l'arthrose, du colostrum en complément alimentaire, ou encore des antioxydants comme le pycnogénol extrait du marc de raisin.

- http://www.pills.com.hk/celebrex.htm

- http://www.nutrition-city.com/antiaging.html

- http://www.health-n-nutrition.com/miracle.html

\section{- Des ouvrages}

Le site amazon.com propose de multiples ouvrages sur le sujet:

$\rightarrow$ Age Erasers for Men : Hundreds of Fast and Easy Ways to Beat the Years by Doug Dollemore, Mark Giuliucci

$\rightarrow$ Age Erasers for Women : Actions You Can Take Right Now to Look Younger and Feel Great by Prevention Magazine Health Books

$\rightarrow$ The Anti-Aging Hormones : That Can Help You Beat the Clock : Benefits and Dangers of Melatonin, Human Growth Hormone DHEA, Oestrogen, Testosterone by Ruth Winter

$\rightarrow$ DHEA...: The Fountain of Youth Discovered by Alan Pascal, Ana Pasca

$\rightarrow$ Fountains of Youth : How to Live Longer and Healthier by Sebastian Orfali, John A. Mann, Ward Dean 
$\rightarrow$ The Ginseng Book : Nature's Ancient Healer by Stephen Fulder

$\rightarrow$ Life Extension : A Practical Scientific Approach by Durk Pearson, Sandy Shaw

$\rightarrow$ Resetting the Clock : 5 Anti-aging hormones that are revolutionising the quality and length of life by Elmer Cranton, William Fryer.

\section{Les stimulants sexuels}

Les internautes pourront trouver aphrodisiaques, stimulants et toniques sexuels, du bois bandé à faire macérer dans du vieux rhum au célèbre petit losange bleu du laboratoire Pfizer, en passant par les cocktails à base de yohimbine ou de ginseng sur de très nombreux sites :

- Le sildenafil, les meilleurs prix, plus d'une cinquantaine d'adresses dont :
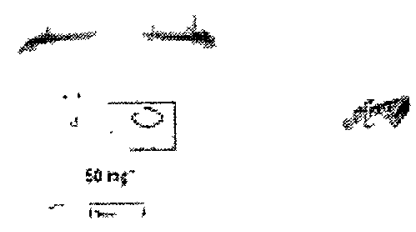

- http://viagra.lovelane.com/

- http://www.pills.com.hk/viagra.htm

- http://www.thepillbox.com/buyviagra.htm

- http://viagra.sh/viagrasearch/5.html

- http://viagra.au.com/

- http://www.onlinehere.com/viagra/index.htm

\section{- La yohimbine}

- http://www.click-here-now.com/drugs/yohimbe.htm

- http://www.feral.org/vitality/active.htm

\section{- Le ginseng}

- http://www.prices-power.com/purple.htm

- http://www.ionnet.com/ginseng.html

\section{- et le bois bandé}

- http://www.xprod.com/xprod-fr.html

Le site amazon.com propose des ouvrages pour utiliser au mieux les produits achetés sur le net :

$\rightarrow$ Better Sex Through Chemistry : A Guide to the New Prosexual Drugs and Nutrients.by John Morgenthaler, Dan Joy

$\rightarrow$ Love Potions : A Guide to Aphrodisiacs and Sexual Pleasures; Cynthia Mervis, M.D. Watson, Angela Hynes $\rightarrow$ The penis book : Use, maintenance and repair by Margaret Gore.

\section{Les dopants de "l'intellect"}

Le dopage pour être plus performant au travail a toujours existé. Mais depuis les Incas qui mâchaient des feuilles de coca pour supporter la fatigue, les produits ont changé et s'achètent aussi sur le Net. Du cadre d' entreprise aux managers de la finance et de la bourse, le primordial est de produire toujours plus, le stress et la pression augmente jusqu'à la prise médicamenteuse et on parle aujourd'hui de dopage des cols blancs.

\section{- Les antidépresseurs}

La consommation d'antidépresseurs, sans être déprimé, juste pour être stimulé est en constante augmentation, en particulier en France.

La fluoxetine (PROZAC $®$ ), l'antidepresseur qui permet de se sentir "better than well" s'achète aux adresses suivantes :

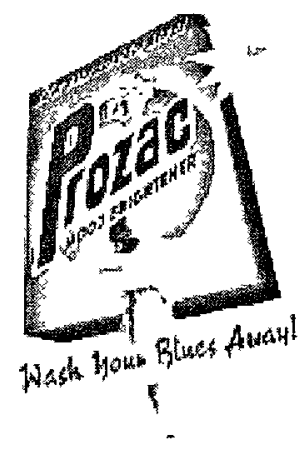

- http://www.pills.com.hk/Prozac_english.htm

- http://www.epills.com

- http://www.bigcapsule.com/prozac.htm

- http://www.insitewebdesign.com/rxusa/prozac

- http://www.click-here-now.com/drugs/ prozacprescriptiondrugs.htm

D'autres antidépresseurs et en particulier inhibiteurs de la recapture de la sérotonine ainsi que des anxiolytiques peuvent se trouver à :

- http://www.ionnet.com/5-htp.html

- http://www.epills.com

- http://www.feral.org/vitality/active.htm

\section{- Les psychostimulants}

Les dérivés des phenethylamines, apparentés aux amphétamines comme l'Ephédrine ou la Pseudoephedrine, la ritaline, le modafinil pour rester éveillé $60 \mathrm{~h}$ d'affilée comme le firent les militaires de la guerre du golfe (sic Internet), des gélules de caféine 
sont à la portée de l'Internaute à :

- http://www.feral.org/vitality/active.htm

- http://www.pricespower.com/energizers

- http://www.click-here-now.com/drugs/ritalinpres criptiondrugs.htm

- http://www.pricespower.com/energizers.htm

\section{- Le GHB et la melatonine}

Comme l'abus de psychostimulants peut entraîner des troubles du sommeil, quelques sites proposent des solutions avec le GHB et la melatonine

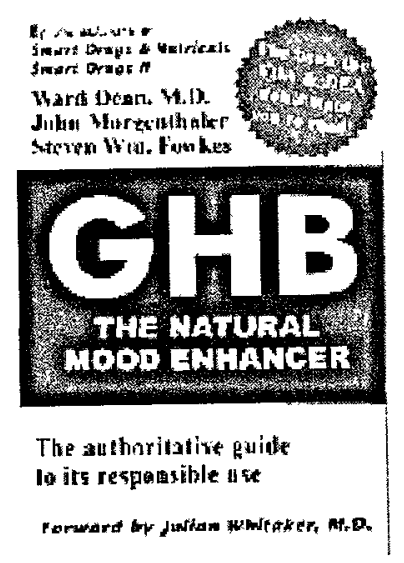

- http://www.feral.org/vitality/active.htm

- http://www.lycaeum.org/drugs/GHB/ghbsynth.html

- http://www.melatonin.com/home.html

- http://www.ounceofprevention.com/wow/order/ melatono.htm

- http://www.pricespower.com/melorder.htm

\section{- Les bêta-bloquants}

Pour lutter contre le trac, augmenter la concentration et la précison du geste, propranolol, acebutolol, atenolol et autres sont en vente à :

- http://www.feral.org/vitality/active.htm

- http://www.epills.com/store/rxbrowse

\section{Le dopage animal}

Le dopage concerne également l'animal. Des sites proposent ainsi des produits à l'intention du meilleur ami de l'homme :

- http://www.equinevetnet.com/

\section{III - Les prix, le paiement, la livraison, les assurances.}

Les prix sont en règle générale très attractifs, voire moins élevés que dans les circuits de vente classiques. Certains sites proposent le remboursement de la diffé- rence si vous trouvez moins cher ailleurs ! Le paiement se fait par carte bancaire, Visa, Mastercard, American Express et il est sécurisé. La livraison se fait par transporteur ou par voie postale, sous forme d'un ou plusieurs colis les plus discrets possibles et sans identification du vendeur ou du contenu. Le délai assuré très rapide dépend de la proximité et du pays d'origine du vendeur.

Le plus surprenant est l'assurance que proposent certains sites pour la livraison : le vendeur ne connaissant pas forcément la législation en vigueur dans le pays de l'acheteur, le produit peut-être bloqué en douane ; il peut également être volé. Une assurance pour le remboursement du produit est ainsi proposée pour quelques dollars de plus sur le site :

- http://www.feral.org/vitality/insurance.htm

\section{IV - Les vendeurs - Mise en garde sur la réglementa- tion - Alerte aux contrefa- çons.}

Les produits pour le dopage sportif (hormis certains stéroïdes anabolisants bannis des pratiques sportives officielles),les produits "anti-aging", aphrodisiaques, plantes, vitamines et minéraux se trouvent principalement sur des sites Américains ou Canadiens. Les stéroïdes et les médicaments peuvent être achetés dans des officines Thaillandaises, Coréennes ou Mexicaines où la législation sur ces produits est plus floue (ou encore sur des sites Anglais (Gibraltar), dont l'officine est à Taiwan !)

La plupart des sites demandent à l'acheteur un engagement écrit pour s'assurer de son âge, qu'il commande pour son usage personnel, pour moins de 3 mois et avec l'accord de son médecin, qu'il connaît la législation du pays de résidence quant à l'importation des substances. Le vendeur décline toute responsabilité relative à un problème réglementaire tout en proposant une assurance quant à la non livraison !

- http://www.feral.org/vitality/legalnotes.htm

- http://www.feral.org/vitality/warnings.htm

Enfin, et en particulier pour les stéroïdes, il existe des contrefaçons, même pour des prođuits estampillés par de grandes firmes pharmaceutiques. Le site PHARMASIA propose de lutter contre en déclarant celles-ci sur une adresse E-mail et en fournissant la liste d'une quarantaine de substances falsifiées.

- http://myhome.shinbiro.com/ pharmasi/ stopfakes.html 


\section{Conclusion}

Se doper sur Internet est effectivement un jeu de souris. Muni d'une carte de crédit et d'une bonne connaissance de la langue anglaise, l'internaute vaillant, les sites étant pléthoriques, trouvera son bonheur avec une liste extravagante de produits. Cette liste impressionnante voire inquiétante intéresse la pratique sportive et le développement musculaire mais aussi la lutte contre le vieillissement, le dopage sexuel et intellectuel ainsi que le dopage animal.

L'internaute pourra également trouver sur le web des conseils pour choisir et utiliser les produits selon la compétition sportive, les schémas posologiques, comment prévenir les effets secondaires, de nombreux témoignages, de multiples ouvrages à acheter et même des listes et descriptions de produits falsifiés.

Le paiement par carte de crédit est maintenant courant et sécurisé par Internet, les prix très attractifs et des assurances peuvent être prises quant à une non réception éventuelle de la commande.

En contrepartie, l'acheteur n'a aucune garantie sur la qualité du produit, même sous l'étiquette d'une grande firme pharmaceutique comme en témoigne la liste des contrefaçons. Il lui faudra être patient pour chercher les sites dont les adresses changent fréquemment.

On ne peut qu'attirer l'attention sur le danger que représente ce marché parallèle de produits classés en France sur la liste II des substances vénéneuses, voire sur la liste I des substances toxiques et qui sont mis à la portée de l'Internaute sans aucun contrôle médical, ni surveillance des abus ou détournement d'usage éventuels. 